\title{
EFFICIENCY AND SCALING \\ OF CURRENT DRIVE AND REFUELLING \\ BY SPHEROMAK INJECTION INTO A TOKAMAK
}

\author{
M.R. BROWN, P.M. BELLAN \\ California Institute of Technology, \\ Pasadena, California, \\ United States of America
}

\begin{abstract}
The first measurements of current drive (refluxing) and refuelling by spheromak injection into a tokamak are discussed in detail. The current drive mechanism is attributed to the process of helicity injection, and refuelling is attributed to the rapid incorporation of the dense spheromak plasma into the tokamak. After an abrupt increase (up to $80 \%$ ), the tokamak current decays by a factor of three because of plasma cooling caused by the merging of the relatively cold spheromak with the tokamak. The tokamak density profile peaks sharply because of the injected spheromak plasma ( $\mathrm{n}_{\mathrm{e}}$ increases by a factor of six) and then becomes hollow, suggestive of an interchange instability. Also discussed is the energy efficiency of spheromak injection current drive and the scaling of this process to larger machines. Refuelling by spheromak injection appears to be a viable scheme for larger machines. However, refluxing by spheromak injection is limited by geometrical and electrical efficiencies (both about 10\%) as well as a high repetition rate requirement.
\end{abstract}

\section{INTRODUCTION}

A spheromak is a force free toroidal magnetofluid equilibrium having comparable toroidal and poloidal magnetic fluxes, with no material linking the centre of the torus [1]. Magnetic helicity is a measure of linked flux, and the formation and sustainment of spheromaks has been explained in terms of electrostatic helicity injection [2]. It has been postulated [3] that since magnetic helicity is a scalar, additive and nearly conserved quantity in resistive MHD (even in the presence of turbulent tearing $[4,5]$ ), the merging of an externally injected spheromak with a tokamak will yield a tokamak of higher helicity content, provided the initial spheromak and tokamak discharges have the same sign of helicity. Since helicity is related to field aligned current and the tokamak toroidal field is fixed by external coils, a tokamak with higher helicity content has higher toroidal current. It has also been suggested that spheromak injection might provide a means of refuelling a tokamak as an alternative to pellet fuelling. Several theoretical models $[3,6,7]$ have been proposed to predict the mechanism whereby an injected spheromak would slow down and be trapped within a tokamak discharge, thereby depositing helicity and density, but until recently there has been no experimental verification of the concept.

We provide here a detailed discussion of the first experimental demonstration of tokamak current drive (refluxing) and refuelling by spheromak injection into the tokamak $[8,9]$. The experiment (see Fig. 1) was performed on Caltech's ENCORE tokamak $(R=0.38 \mathrm{~m}$, $\mathrm{a}=0.12 \mathrm{~m}, \mathrm{I}_{\mathrm{p}}=2 \mathrm{kA}, \mathrm{B}_{\text {tor }}=0.07 \mathrm{~T}$ ), with a spheromak $\left(r=0.04 \mathrm{~m}, \Phi_{\text {gun }}=0.4 \mathrm{mWb}\right.$ and $\left.I_{\text {gun }}=80 \mathrm{kA}\right)$ injected by a magnetized coaxial plasma gun. The injection of a spheromak results in a number of phenomena. (1) We observe an initial increase of up to $80 \%$ in tokamak plasma current (from 2.0 to $3.6 \mathrm{kA}$ ) upon injection of a spheromak only when both spheromak and tokamak have the same sign of helicity. The sign of helicity is defined as positive (right handed) if current flows parallel to the magnetic field and negative (left handed) if it flows anti-parallel. A more typical increase in current is $30 \%$. If the spheromak and the tokamak have different signs of helicity, the tokamak current drops by about $20 \%$ in a typical case. The

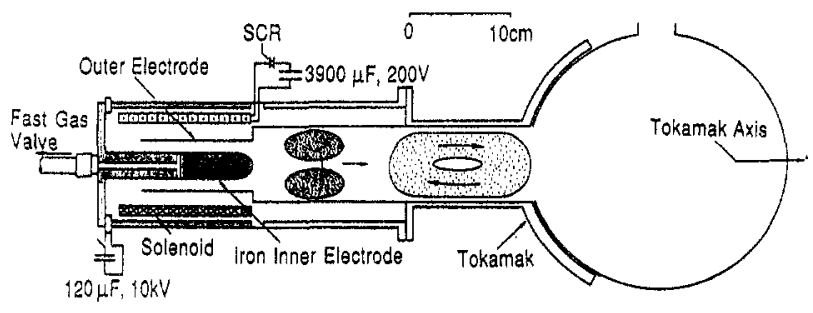

FIG. 1. Schematic of experimental set-up showing the topology of the injected spheromak. Typical parameters for the gun are $\Phi_{\text {gun }}=0.5 \mathrm{mWb}$ and $I_{\text {gun }}=100 \mathrm{kA}$, and for the discharge $n_{\text {sph }}=10^{2 I} \mathrm{~m}^{-3}$ and $\mathrm{v}=3 \times 10^{4} \mathrm{~m} / \mathrm{s}$. 
initial increase (decrease) in current is accompanied by a sharp decrease (increase) in loop voltage, and the increase in tokamak helicity is consistent with the helicity content of the injected spheromak. (2) After the initial increase ( $\cong 30 \mu$ s after injection) we observe a decline in current to a lower level because of a sudden cooling of the tokamak plasma from $12 \mathrm{eV}$ to $4 \mathrm{eV}$ and the addition of impurities. (3) We observe an increase in tokamak central density by a factor of six. The density profile becomes peaked, which is indicative of fuelling on axis, followed by what appears to be an interchange instability which flattens the profile.

The paper is organized as follows. In Section 2, we discuss important theoretical concepts including magnetic helicity and the maximum expected efficiency of spheromak injection current drive. In Section 3, we briefly describe the experimental apparatus. The results of spheromak injection current drive and refuelling are discussed in Section 4; in particular, the variation of injected tokamak helicity with spheromak gun voltage and flux is presented. Finally, in Section 5, we discuss the scaling of spheromak injection to larger tokamaks.

\section{THEORETICAL CONCEPTS}

\subsection{Relationship between helicity and current drive}

Magnetic helicity is formally defined as

$K=\int \vec{A} \cdot \vec{B} d^{3} x$

where the integral is performed within a closed, perfectly conducting boundary. Helicity has the units of magnetic flux squared and can be thought of as a measure of the linked flux within a closed boundary [10]. It can also be viewed as a volume averaged measure of the 'twistedness' of the magnetic field lines in a closed system. Because of the arbitrariness of the vector potential $\vec{A}$, there can be ambiguities in the definition of helicity, depending on the choice of gauge at the boundary. If the boundary is chosen to be perfectly conducting (so that the boundary is a magnetic surface), then these ambiguities are largely resolved.

It can be shown [11] that the change in helicity resulting from a change in gauge, $\overrightarrow{\mathbf{A}} \rightarrow \overrightarrow{\mathbf{A}}+\nabla \xi$, is

$$
\begin{aligned}
\Delta \mathrm{K} & =\int \nabla \xi \cdot \overrightarrow{\mathrm{B}} \mathrm{d}^{3} \mathrm{x} \\
& =\int \xi \overrightarrow{\mathrm{B}} \cdot \overrightarrow{\mathrm{d}} \mathrm{s}
\end{aligned}
$$

If the boundary is a perfect conductor, then it is a magnetic surface, $\overrightarrow{\mathrm{B}} \cdot \overrightarrow{\mathrm{ds}}=0$, and $\mathrm{K}$ is gauge invariant.

The relationship between helicity and field aligned current is depicted in Fig. 2. If we imagine a closed cylindrical volume containing plasma in a uniform axial magnetic field $\mathbf{B}_{\text {tor }}$, then helicity can be injected into the volume by applying a potential difference across the end caps. The potential difference drives an axial current which generates a magnetic field $B_{\text {pol }}$, which encircles the pre-existing axial field. The helicity that is generated by the field aligned current is roughly $\mathrm{K} \cong \Phi_{\text {tor }} \Phi_{\text {pol }}$, where $\Phi$ is the flux. The rate at which helicity is injected is given [2] by $\dot{K}=2 \mathrm{~V} \Phi_{\text {tor }}$, where $\mathrm{V}$ is the applied potential difference. Helicity injection is then ultimately balanced by Ohmic dissipation.

We can see from Fig. 2 that if the field aligned current is parallel (anti-parallel) to the axial field, then the resultant field lines have a right handed (left handed) pitch. Because helicity is a scalar, we can ascribe the sign of the helicity to the sense of the pitch. We will hereafter refer to systems with current parallel to the magnetic field as having positive or right handed helicity, and to systems with current anti-parallel to the magnetic field as having negative or left handed helicity. Another ramification of the scalar nature of helicity is that it is additive. It can be shown [11] that in a simply

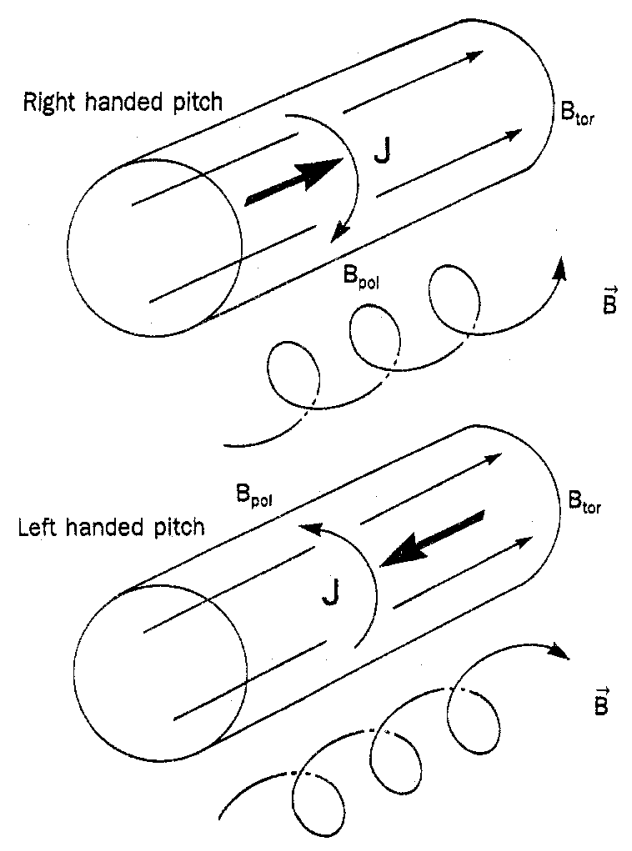

FIG. 2. Helicity related to field aligned current. The current density $J$, flowing parallel to $B_{\text {tor }}$ generates a linking field, $B_{p o l}$, so that a typical field line has a right handed pitch. Anti-parallel current generates field lines of left handed pitch. 
connected volume, the total helicity can be expressed as the algebraic sum of the separate helicities of the subvolumes as long as the background magnetic fields are axisymmetric with the volume. In a non-simply connected volume such as a tokamak, the flux linking the hole in the torus must be considered. We take this linking flux into account in our experiments.

Helicity and magnetic energy are conserved quantities in ideal MHD and decay on a resistive time-scale in dissipative systems [12]. However, magnetic energy can be readily dissipated by magnetic turbulence. The remarkable feature of magnetic helicity is that it is nearly conserved even in the presence of high magnetic Reynolds number turbulence. This has been suggested by Taylor $[4,5]$ and subsequently verified experimentally in reversed field pinches [13] and spheromaks [2].

In dissipative MHD, helicity and magnetic energy decay according to

$\dot{\mathrm{K}} \cong-2 \int \overrightarrow{\mathrm{E}} \cdot \overrightarrow{\mathrm{B}} \mathrm{d}^{3} \mathrm{x} \cong-2 \eta \int \overrightarrow{\mathrm{J}} \cdot \overrightarrow{\mathrm{B}} \mathrm{d}^{3} \mathrm{x}$

$\dot{W} \cong-\int \vec{E} \cdot \vec{J} d^{3} x \cong-\eta \int \vec{J} \cdot \vec{J} d^{3} x$

since the large scale (DC) magnetic field is simply proportional to J. Note that Eq. (3) is a direct result of Faraday's law and the MHD Ohm's law; Eq. (4) is Poynting's theorem. It can be shown [5] that in the presence of small scale turbulent fluctuations of wave number $k$, the turbulent dissipation is expressible in Fourier representation:

$\dot{\mathrm{K}} \cong-2 \eta \sum \mathrm{kB}_{\mathrm{k}}^{2}$

$\dot{\mathrm{W}} \cong-\eta \sum \mathrm{k}^{2} \mathrm{~B}_{\mathrm{k}}^{2}$

so that small scale (large $\mathrm{k}$ ) turbulence is much more effective at dissipating energy than it is at dissipating helicity. We see that at large scales (and low frequencies), both helicity and energy decay at nearly the same rate. Actually, Eq. (5) is an upper bound on the helicity dissipation since we have assumed that $\vec{J}$ and $\vec{B}$ are aligned $(\cos \theta=1)$. If $\vec{J}$ and $\vec{B}$ are anti-aligned, then Eq. (5) can become a helicity source.

The preferential decay of energy compared to helicity has also been discussed in the context of MHD turbulence. Frisch et al. [14] have suggested that threedimensional turbulent MHD flows can lead to an inverse cascade of magnetic helicity generating large scale magnetic structure. Montgomery et al. [15] have discussed in detail the role of the ratio of helicity to energy, $\mathrm{K} / \mathrm{W}$, in the relaxation of turbulent flows.
Spheromak injection current drive can now be described in the following way. A high helicity content spheromak (of a particular helicity sign) is rapidly injected into a tokamak. The spheromak then reconnects with the tokamak via turbulence in a tearing time (slow reconnection) or an Alfvén time (fast reconnection), both of which are short compared with the resistive diffusion time. Although the turbulent merging process rapidly dissipates energy (from Eq. (6)), the helicity of the system is nearly conserved and becomes the simple sum of the spheromak and tokamak helicities. If the spheromak and the tokamak had the same helicity sign, then the merging process would yield a tokamak of higher helicity. Since the toroidal flux of the tokamak is fixed with high inductance external coils, an increase in tokamak helicity is tantamount to an increase in tokamak poloidal flux (or toroidal current).

Figure 3 shows schematically the process of spheromak injection current drive [16]. We can view the high helicity content spheromak as a tightly coiled spring and the relatively low helicity content tokamak as a loosely coiled spring. The tightly coiled spring of the spheromak is rapidly merged with the loosely coiled spring of the tokamak. This reconnection process can be pictured as cutting the tokamak spring, inserting the spheromak spring and welding the joints. If the spheromak spring and the tokamak spring had the same sense of pitch (say both right handed), then, when the spring tensions relaxed, we would have a tokamak spring of

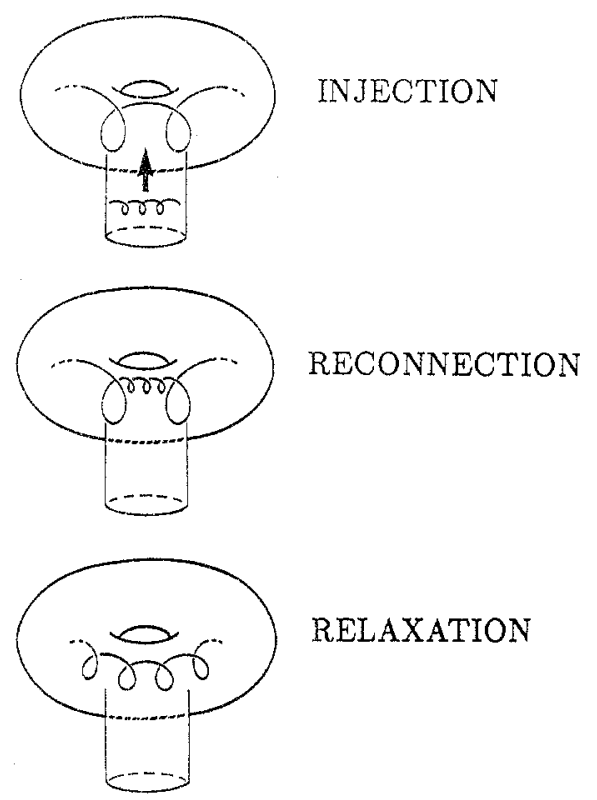

FIG. 3. Spheromak injection current drive analogous to merging springs of the same pitch. 
higher pitch. The higher pitched spring is analogous to tokamak field lines of higher pitch. Higher pitched field lines correspond to higher poloidal flux, which is the same as higher toroidal current.

It is also worth noting that there are other magnetofluid systems in which sustainment is better understood by direct replenishment of magnetic flux rather than by driving a current. The magnetic energy of the Earth's magnetosphere is increased not by direct current drive (although this is a consequence) but by the addition of flux from the solar wind through reconnection. A magnetic field previously entrained in the solar wind reconnects with the Earth's magnetic field, thereby adding magnetic energy.

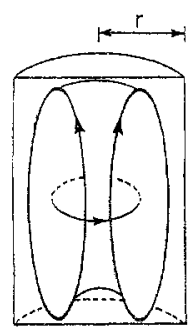

(a)

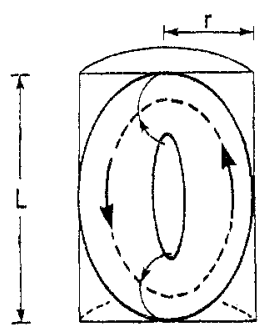

(b)
FIG. 4. Spheromak geometries: (a) axisymmetric and (b) non-axisymmetric.

\subsection{Expressions for spheromak and tokamak helicity}

From the standpoint of helicity accounting, it is useful to have expressions for spheromak and tokamak helicity in terms of easily measured quantities. The helicity content of a magnetized plasma volume can be estimated in a number of ways.

First, the formal definition $\left(\mathrm{K}=\int \overrightarrow{\mathrm{A}} \cdot \overrightarrow{\mathrm{B}} \mathrm{d}^{3} \mathrm{x}\right)$ can be approximated by $K_{\text {sph }} \cong \alpha \Phi_{\text {tor }} \Phi_{\text {pol }}$, where $\alpha$ is a constant of order unity. Therefore, the helicity can be approximated by calculating the linked flux product. For the case of two separate, discrete, singly linked fluxes [10], $\alpha=2$; for the case of an axisymmetric spheromak in a cylindrical flux conserver [17], $\alpha$ is close to unity.

A spheromak in a cylindrical vessel can be oriented either axisymmetric with the cylinder or tilted $[18,19]$. For the case of an axisymmetric spheromak (Fig. 4(a)), the toroidal flux passes through an ellipse of length $L$; the linking poloidal flux threads through a circle of radius $r / 2$ (where $r$ is the radius of the cylinder). We can therefore estimate

$$
\begin{gathered}
\mathrm{K}_{\mathrm{sph}} \cong \Phi_{\mathrm{tor}} \Phi_{\mathrm{pol}} \cong\left[\mathrm{B}_{\mathrm{avg}} \pi(\mathrm{L} / 2)(\mathrm{r} / 2)\right] \\
\times\left[\mathrm{B}_{\mathrm{avg}} \pi \mathrm{r}^{2} / 4\right] \cong \mathrm{B}_{\mathrm{avg}}^{2} \pi^{2} \mathrm{Lr}^{3} / 16
\end{gathered}
$$

where $B_{\text {avg }}$ is the volume averaged magnetic field measured in the spheromak equilibrium. For a tilted, non-axisymmetric spheromak (Fig. 4(b)), the toroidal flux passes through one half of the circular crosssection; the linking poloidal flux threads an ellipse of minor radius $r / 2$ and major radius $L / 4$. In this case,

$\mathrm{K}_{\text {sph }} \cong\left[\mathrm{B}_{\text {avg }} \pi \mathrm{r}^{2} / 2\right] \times\left[\mathrm{B}_{\text {avg }} \pi \mathrm{Lr} / 8\right] \cong \mathrm{B}_{\text {avg }}^{2} \pi^{2} \mathrm{Lr}^{3} / 16$

which is the same result as that in the axisymmetric case. We now have a robust, approximate expression for spheromak helicity in cylindrical flux conservers. The helicity content of the spheromak that ultimately moves into the tokamak can be estimated from measurements performed on the spheromak in the tokamak vessel without tokamak plasma. From extensive magnetic probe measurements performed in the tokamak vacuum vessel [20] we typically find $B_{\text {avg }} \cong 0.02 \mathrm{~T}, \mathrm{r}=0.12 \mathrm{~m}$ and $\mathrm{L} \cong 4 \mathrm{r}$, so that $\mathrm{K}_{\mathrm{sph}}=3 \times 10^{-7} \mathrm{~Wb}^{2}$ from the above expressions.

Second, it has been shown by Taylor $[4,5]$ that if the magnetic energy of a magnetofluid, $W=\int B^{2} / 2 \mu_{0} d^{3} x$, is minimized subject to the constraint of constant helicity $\mathrm{K}$, then the equilibrium magnetic states in a perfectly conducting boundary satisfy the force free condition $\nabla \times \vec{B}=\lambda \vec{B}$, where $\lambda$ is the Lagrange multiplier in the minimization calculation. In this interpretation, we can write $\lambda=2 \mu_{0} \mathrm{~W} / \mathrm{K}$, where $\lambda$ has dimensions of (length) $)^{-1}$ and varies inversely with the size of the flux conserver $[18,19]$.

We can therefore make another estimate by noting [2] that the energy per unit helicity of the spheromak equilibrium, $2 \mu_{0} \mathrm{~W}_{\mathrm{mag}} / \mathrm{K}_{\mathrm{sph}}$, is equal to $\lambda$. Here, $\mathrm{W}_{\text {mag }}=\left(\mathrm{B}_{\text {avg }}^{2} / 2 \mu_{0}\right) \pi \mathrm{r}^{2} \mathrm{~L}$ is the spheromak magnetic energy in a cylindrical flux conserver and $\lambda$ is the eigenvalue of the equation of the force free state, $\nabla \times \overrightarrow{\mathrm{B}}=\lambda \overrightarrow{\mathrm{B}}$. Again, for an axisymmetric spheromak in a cylindrical flux conserver we have (see Ref. [19]) $\lambda=\left(\mathrm{k}_{\mathrm{z}}^{2}+\mathrm{k}_{\mathrm{r}}^{2}\right)^{1 / 2}$, where $\mathrm{k}_{\mathrm{z}}=\pi / \mathrm{L}$ and $\mathrm{k}_{\mathrm{r}}=3.83 / \mathrm{r}$. From the measurements discussed above, we find $\lambda=32 \mathrm{~m}^{-1}$, from which we obtain the same result, $\mathrm{K}_{\mathrm{sph}}=3 \times 10^{-7} \mathrm{~Wb}^{2}$. We have performed a more careful calculation of $\lambda$ from a fit of data to a simple model [20] and have found a similar result.

Third, we can estimate the helicity content of the spheromak by first noting that helicity is generated by 
the coaxial magnetized plasma gun at a rate (see Ref. [2]) $\dot{\mathrm{K}}=2 \mathrm{~V}_{\text {gun }} \Phi_{\text {gun }}$, where $\mathrm{V}_{\text {gun }}$ is the voltage that develops between inner and outer electrodes, and $\Phi_{\text {gun }}$ is the magnetic flux imbedded in the central electrode. Note that $\mathrm{V}_{\text {gun }}$ is determined by dynamic gun impedance and is usually significantly less than the voltage on the capacitor bank. We find that

$\mathrm{K}_{\text {initial }} \cong \int 2 \mathrm{~V}_{\text {gun }} \Phi_{\text {gun }} \mathrm{dt}$

In our case, we find $\mathrm{K}_{\text {initial }} \cong 4 \times 10^{-6} \mathrm{~Wb}^{2}$ for typical values of $\mathrm{V}_{\text {gun }}=1 \mathrm{kV}$ and $\Phi_{\text {gun }}=0.4 \mathrm{mWb}$, and the time during which the voltage is applied is $\Delta \mathrm{t} \cong 5 \mu \mathrm{s}$. We make the further observations that (1) the magnetic e-folding time of our spheromaks is $10 \mu \mathrm{s}$, so that the helicity decay time, $\tau_{\mathrm{K}} \cong \tau_{\mathrm{B}} / 2$, is about $5 \mu \mathrm{s}$, and (2) that the velocity of our spheromaks is about $3 \mathrm{~cm} / \mu \mathrm{s}$. Thus, we can infer that the initial helicity content of our spheromak decays by about two e-folding times while traversing the $30 \mathrm{~cm}$ between gun and tokamak. We find by this calculation $\mathrm{K}_{\mathrm{sph}}=5 \times 10^{-7} \mathrm{~Wb}^{2}$, consistent with the other two methods.

It is worth pointing out that the helicity injection effect was not observed in other experiments [21] where the helicity decay time was $20 \%$ shorter. In these early experiments the helicity decay time was shorter because of impurities from plastic rather than ceramic insulators. In addition, edge dissipation effects may have played a significant role. It appears that if the helicity decay time is similar to or short compared with the spheromak transit time from gun to tokamak, then no helicity injection effect will be seen. For a hot, rapidly moving spheromak, the resistive loss of helicity in transit to the tokamak should be small. Note that neither helicity nor magnetic flux is lost during a spheromak tilt, so details of spheromak motion should not affect the helicity injection results [20].

Finally, the tokamak helicity can be estimated by $\mathrm{K}_{\text {tok }} \cong \Phi_{\text {tor }} \Phi_{\text {pol }}$, so we obtain (for a flat current profile) $\mathrm{K}_{\text {tok }} \cong\left[\mathrm{B}_{\text {tor }} \pi \mathrm{a}^{2}\right] \times\left[\mu_{0} \mathrm{I}_{\mathrm{p}} \mathrm{R} / 2\right]=\mu_{0} \pi \mathrm{B}_{\text {tor }} \mathrm{I}_{\mathrm{p}} \mathrm{a}^{2} \mathrm{R} / 2$

Note that $\mathrm{K}_{\text {tok }}$ depends linearly on both $\mathrm{B}_{\text {tor }}$ and $\mathrm{I}_{\mathrm{p}}$ and also scales linearly with the tokamak volume. If the current profile is peaked with a parabolic profile, then $\mathrm{K}_{\text {peaked }}=\frac{3}{2} \mathrm{~K}_{\text {flat }}$.

\subsection{Theoretical efficiency of spheromak injection current drive}

We noted previously that minimizing the magnetic energy $W$ of a magnetofluid subject to the constraint of constant helicity $\mathrm{K}$ results in the force free condition $\nabla \times \overrightarrow{\mathrm{B}}=\lambda \overrightarrow{\mathrm{B}}$. Here, $\lambda=2 \mu_{0} \mathrm{~W} / \mathrm{K}$ is interpreted as a measure of the inverse of the scale size of the system. The eigenvalue $\lambda$ can also be interpreted as a ratio of current density to magnetic field, $\lambda=\mu_{0} \mathrm{~J} / \mathrm{B}$. If we multiply both the numerator and the denominator of this expression by a suitable area, then we have $\lambda=\mu_{0} \mathrm{I} / \Phi$. In this interpretation, $\lambda$ is a measure of the discharge current necessary in the spheromak gun in order to overcome the magnetic gun flux [20, 22]. This threshold varies inversely with the radius of the gun.

Barnes et al. $[2,22]$ have shown that the equation representing tokamak helicity balance

$$
\frac{\partial \mathrm{K}_{\mathrm{tok}}}{\partial \mathrm{t}}=\frac{-\mathrm{K}_{\mathrm{tok}}}{\tau_{\mathrm{K}}}+2 \mathrm{~V}_{\mathrm{gun}} \Phi_{\mathrm{gun}}
$$

can be rewritten as an energy balance equation, where $\tau_{\mathrm{K}}$ is the helicity decay time. If we note that $\lambda_{\text {tok }}=2 \mu_{0} \mathrm{~W}_{\text {tok }} / \mathrm{K}_{\text {tok }}$ and $\lambda_{\text {gun }}=\mu_{0} \mathrm{I}_{\text {gun }} / \Phi_{\text {gun }}$, then

Eq. (11) can be expressed as

$\frac{\partial \mathrm{W}_{\mathrm{tok}}}{\partial \mathrm{t}}=\frac{-\mathrm{W}_{\mathrm{tok}}}{\tau_{\mathrm{K}}}+\epsilon \mathrm{I}_{\mathrm{gun}} \mathrm{V}_{\mathrm{gun}}$

where $\epsilon=\lambda_{\text {tok }} / \lambda_{\text {gun }}$ and we assume that perturbation to the magnetic geometry of the tokamak by the spheromak is small, i.e. $\partial \lambda_{\text {tok }} / \partial t \cong 0$. So, Eq. (11) describes tokamak helicity balance where helicity is generated by the spheromak gun at a rate $2 \mathrm{~V}_{\text {gun }} \Phi_{\text {gun }}$ and dissipated in the tokamak at a rate $-\mathrm{K}_{\mathrm{tok}} / \tau_{\mathrm{K}}$. Equation (12) describes the tokamak energy balance where poloidal magnetic field energy is provided by the gun at a rate $\mathrm{I}_{\text {gun }} \mathrm{V}_{\text {gun }}$ derated by the factor $\epsilon$. Energy at the largest scale is dissipated at a rate $-\mathrm{W}_{\text {tok }} / \tau_{\mathrm{K}} \cong-\mathrm{W}_{\text {tok }} / \tau_{\mathrm{W}}$ where the helicity and energy dissipation rates are similar in the large scale, low frequency limit.

Note that the additional power $(1-\epsilon) \mathbf{I}_{\mathrm{gun}} \mathrm{V}_{\mathrm{gun}}$ must be dissipated by turbulence during relaxation to the minimum energy state. As the spheromak plasma merges with the larger tokamak discharge, the new boundary conditions require that the characteristic $\lambda$ should drop. Since $\lambda=2 \mu_{0} \mathrm{~W} / \mathrm{K}$ and since the helicity $\mathrm{K}$ is conserved, the energy $W$ is rapidly dissipated to regulate $\lambda$.

The $\lambda$ factor appropriate for a tokamak can be written (see Ref. [23]) $\lambda_{\text {tok }}=2 / \mathrm{q}_{\mathrm{wall}} \mathrm{R}$, where $\mathrm{q}_{\mathrm{wall}}=\left(\mathrm{B}_{\mathrm{tor}} \mathrm{a}\right) /\left(\mathrm{B}_{\mathrm{pol}} \mathrm{R}\right)$ is the tokamak safety factor and we have used $\mathrm{Eq}$. (10). The precise value of $\lambda_{\text {tok }}$ depends on the shape of the current profile; $\lambda_{\text {tok }}$ increases by $20 \%$ as the current profile peaks from a flat profile to a parabolic one with fixed $I_{p}$. The $\lambda$ factor for the gun can be approximated as $\lambda_{\text {gun }} \cong 4 / r_{\text {gun }}$; the precise coefficient depends on the geometry of the gun $[20,22]$. We therefore find

$\epsilon=\lambda_{\text {tok }} / \lambda_{\text {gun }} \cong\left(2 / q_{\text {wall }} R\right)\left(r_{\text {gun }} / 4\right)=r_{\text {gun }} / 2 q_{\text {wall }} R$ 
This is the maximum efficiency expected for spheromak injection current drive. For a typical tokamak discharge with $\mathrm{q}_{\mathrm{wall}}=3$ and an aspect ratio of 3 we find $\epsilon<5 \%$ (we assume that $r_{\text {gun }}$ can be no larger than the tokamak minor radius). Note that $\epsilon$ is a purely geometrical quantity $\left(\epsilon \propto \mathrm{r}_{\mathrm{gun}} / \mathrm{R}_{\mathrm{tok}}\right)$ so that spheromak injection current drive can be within a factor $\epsilon$ of Ohmic current drive. The factor $\epsilon$ merely reflects the inefficiency of the process of relaxation. Relaxation is the process where spheromak energy is dissipated rapidly by turbulence (while helicity is conserved) as the spheromak moves into a region of lower characteristic $\lambda$ (i.e. larger size).

The conventional RF current drive figure of merit or 'efficiency' is defined [24] as

$\eta_{\mathrm{CD}}=\frac{\mathrm{nI}_{\mathrm{p}} \mathrm{R}}{\mathrm{P}}$

where $\mathrm{P}$ is the steady state RF power applied to the tokamak of major radius R. We can see from Eq. (14) that the conventional RF current drive 'efficiency' has a factor of an inverse voltage and therefore favours low voltage, low resistance, hot plasmas. In particular, if we assume in Eq. (14) that $\mathrm{P}=\mathrm{mv}^{2} \nu$, where $\mathrm{v}$ is the velocity of the current carrying electrons and $\nu$ is the collision frequency, and that $\nu \propto \mathrm{v}^{-3}$, then $\eta_{\mathrm{CD}} \propto \mathrm{v}^{2}$. In the case of fast current carriers (for example, lower hybrid current drive with $v_{\mathrm{car}} \gg \mathrm{v}_{\mathrm{th}}$ ), the efficiency defined in this way can be very high. Therefore, Eq. (14) is not the appropriate expression for spheromak injection current drive. We therefore seek a more appropriate figure of merit for spheromak injection current drive.

In steady state spheromak injection current drive, we can define an efficiency from Eq. (12):

$\eta_{\mathrm{sph}}=\frac{\mathrm{W}_{\mathrm{tok}}}{\mathrm{I}_{\text {gun }} \mathrm{V}_{\mathrm{gun}}}=\epsilon \tau_{\mathrm{K}}$

so that the stored energy in the tokamak poloidal fields is replenished by the gun power in a helicity decay time (again, derated by the factor $\epsilon$ ). This definition of efficiency has units of time and also improves with hotter plasmas, but it is more suited to spheromak injection current drive since it quantifies a process of energy transfer from the spheromak gun to the tokamak poloidal field. In order to reflux a tokamak, the gun must be fired once every $\epsilon \tau_{\mathrm{K}}$. In other words, the geometrical factor $\epsilon$ is exactly the ratio of the power dissipated by the tokamak magnetic fields $\left(\mathrm{W}_{\mathrm{tok}} \tau_{\mathrm{K}}\right)$ to the power at the spheromak gun $\left(\mathrm{I}_{\mathrm{gun}} \mathrm{V}_{\mathrm{gun}}\right)$. It is in this sense that spheromak injection current drive can be within a factor of $\epsilon$ of Ohmic current drive. Note that the definition of $\eta_{\mathrm{sph}}$ in Eq. (15) is similar to the definition of tokamak 'ramp-up' efficiency [24], $\dot{\mathrm{W}}_{\mathrm{tok}} / \mathrm{P}_{\mathrm{rf}}$, where the kinetic energy of the current carriers is ignored and only the stored energy in tokamak poloidal fields is considered.

We see that efficient current drive by the process of spheromak injection requires that $r_{\text {gun }} / R_{\text {tok }}$ be as large as possible. Spheromaks can also be used to refuel as well as reflux a tokamak. We now show that efficient tokamak refuelling requires that $r_{\text {gun }} / R_{\text {tok }} \ll 1$, which is in conflict with spheromak injection refluxing requirements. The requirement for spheromak penetration into a tokamak can be written $[6,7,20]$ as

$\frac{\rho_{\text {sph }} \mathrm{v}_{\mathrm{sph}}^{2}}{\mathrm{~B}_{\mathrm{tok}}^{2} / \mu_{0}}=\beta_{\mathrm{dyn}}>1$

where $\rho_{\mathrm{sph}}$ and $\mathrm{v}_{\mathrm{sph}}$ are the spheromak density and velocity, respectively. This is merely a statement that the dynamic $\beta$ or the ratio of spheromak kinetic energy to tokamak magnetic energy must exceed unity.

We should point out that the criterion of Eq. (16) can be realized either by forming a dense spheromak and allowing it to drift into the tokamak (as we have done in the experiments described in Section 4) or by accelerating the spheromak with a separate stage, as has been done by the RACE group [25]. In the absence of an acceleration stage, the spheromak velocity is the internal Alfvern velocity. This is because the spheromak velocity results from the internal gun fields pushing on the spheromak density and $\mathrm{v}_{\mathrm{Alf}} \propto \mathrm{B} \rho_{\mathrm{sph}}^{-1 / 2}$. In this case, Eq. (16) reduces to $B_{\mathrm{sph}} / B_{\mathrm{tok}}>1$. The use of an acceleration stage effectively decouples the velocity of the spheromak from internal fields and density.

\section{EXPERIMENTAL APPARATUS}

The spheromak in our experiment (Fig. 1) is formed by a coaxial, magnetized plasma gun similar in design to that used by Alfvén [26] and is described in detail elsewhere $[8,9,20,21]$. An axial $\vec{J} \times \vec{B}$ force accelerates the plasma along the gun barrel until it reaches the magnetic flux, $\Phi_{\text {gun }}$, at the gun muzzle. If the $\vec{J} \times \vec{B}$ force is strong enough to overcome the magnetic tension at the gun muzzle, then a free spheromak forms and propagates away from the gun. As sketched in Fig. 1, the spheromak is observed (using magnetic probes) to tilt in the entrance region between spheromak gun and tokamak [20].

We typically operate with $\Phi_{\text {gun }}=0.3-0.5 \mathrm{mWb}$ and discharge current $I_{\text {gun }}=60-120 \mathrm{kA}$, with a $3.5-7 \mathrm{kV}$ charge on the capacitor. We have found it necessary to operate above a gun threshold, $\lambda_{\text {th }}=\mu_{0} I_{\text {gun }} / \Phi_{\text {gun }}$ 
$\cong 210 \mathrm{~m}^{-1}$, otherwise a free spheromak is not formed [20]. Barnes et al. [2] have made similar observations on the CTX spheromak with an appropriately smaller $\lambda_{\mathrm{th}}$. The spheromak moves from the gun to the tokamak at the local Alfven velocity (about $3 \times 10^{4} \mathrm{~m} / \mathrm{s}$ ). Typical spheromak densities and temperatures are $10^{21} \mathrm{~m}^{-3}$ and $7 \mathrm{eV}$, as measured by a double Langmuir probe in the tokamak vessel directly in front of the gun. The density measurements have been corroborated with a $3 \mathrm{~mm}$ density interferometer (on the opposite side of the tokamak chamber). The tokamak vessel volume is about a factor of 100 larger than the initial spheromak volume, and peak densities of about $10^{19} \mathrm{~m}^{-3}$ are measured with the interferometer. As the spheromak plasma expands to fill the tokamak, its density drops by at least the ratio of spheromak volume to tokamak volume. If there is recombination or other plasma loss, this technique will underestimate the initial spheromak density.

\section{RESULTS}

\subsection{Current drive by spheromak injection}

We are able to generate spheromak and tokamak discharges of either helicity sign. We reverse the helicity sign of the spheromak by changing the direction of the gun flux $\Phi_{\text {gun }}$, and we reverse the helicity sign of the tokamak by reversing the direction of the toroidal field. Figure 5 shows our helicity injection results with all combinations of helicity sign. Figures 5(a) and (c) show the tokamak plasma current $I_{p}$ with injection of a spheromak into a tokamak discharge of the same sign (left into left and right into right, respectively), while Figs 5(b) and (d) show the effect of injecting a spheromak into a tokamak of opposite sign (left into right and right into left, respectively). Note that we are able to change the sign of tokamak helicity and spheromak helicity independently and have found an increase in $I_{p}$ (i.e. helicity) only when tokamak and spheromak have the same helicity sign. When the tokamak and the spheromak have opposite helicity sign, there is a sharp decrease in $I_{p}$ upon spheromak injection.

Operationally, we change the sign of $\mathrm{K}_{\mathrm{sph}}$ by reversing the direction of the stuffing flux (while maintaining the polarity of the electrodes) and we change the sign of $\mathrm{K}_{\mathrm{tok}}$ by reversing the sign of $\mathrm{B}_{0}$ (while maintaining the direction of $I_{p}$ ). For practical reasons, we operate with a negative central electrode on the gun [21]. We have found that gas breakdown is more effective with a negative central electrode. We have performed experi- ments in which the sign of $\mathrm{K}_{\mathrm{tok}}$ was changed by reversing the direction of $I_{p}$ with the same results as noted above, i.e. there is an increase in $I_{p}$ only when tokamak and spheromak have the same helicity sign.

We are able to calculate the increase in tokamak helicity from the increase in tokamak current using Eq. (10). We find $\Delta \mathrm{K}_{\text {tok }} \cong 4.5 \times 10^{-7} \mathrm{~Wb}^{2}$ for $\Delta \mathrm{I}_{\mathrm{p}}=600 \mathrm{~A}$ and $B_{\text {tor }}=0.07 \mathrm{~T}$. We conclude that the increase in tokamak helicity is consistent with the helicity of the injected spheromak estimated in Section 2.2. We therefore see that the current drive effect is consistent in both sign and magnitude with what we expect from helicity injection.

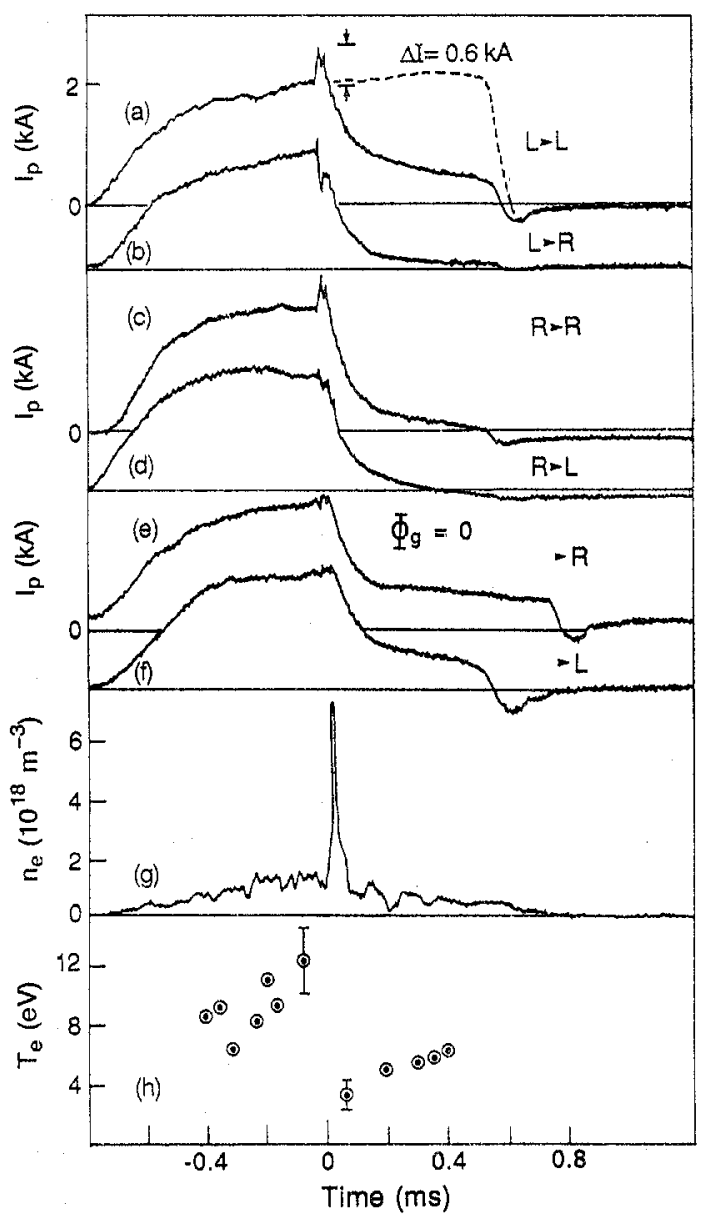

FIG. 5. Time history of a tokamak discharge $\left(B_{\phi}=0.07 \mathrm{~T}\right)$ with spheromak injection $\left(I_{\text {gun }}=80 \mathrm{kA}, \Phi_{\text {gun }}=0.4 \mathrm{mWb}\right)$; time-scale relative to injection; each trace is a single discharge. Plasma current: (a) with a left handed spheromak injected into a left handed tokamak (the dashed line indicates the normal discharge without spheromak injection), (b) left into right, (c) right into right, (d) right into left, (e) Marhsall gun $\left(\Phi_{\text {gun }} \cong 0\right.$ ) injection into left handed tokamak, and (f) Marshall gun injection into right handed tokamak; (g) central electron density and (h) central electron temperature traces from a Langmuir probe. 
We can calculate the expected geometrical efficiency of our experiment from Eq. (13). We found that it was necessary to operate the tokamak at relatively high edge $\mathrm{q}$ in order to provide a stable tokamak discharge that would not be terminated by spheromak injection. Spheromak injection led to a peaked density profile, followed by a rapid hollowing of the profile (see Section 4.2). This effect was mitigated by operating at higher $\mathrm{q}$. We typically operated at $\mathrm{q}_{\mathrm{wall}}=7\left(\mathrm{~B}_{\mathrm{tor}}=0.07 \mathrm{~T}\right.$ and $I_{p}=2 \mathrm{kA}$ ) so that $\lambda_{\text {tok }}=0.75 \mathrm{~m}^{-1}$. Our best spheromak operation occurred at $\lambda_{\text {gun }} \cong 250 \mathrm{~m}^{-1}$, so that $\epsilon=\lambda_{\text {tok }} / \lambda_{\text {gun }} \cong 3 \times 10^{-3}$. Since the stored energy in the capacitor bank was about $1500 \mathrm{~J}$, we would therefore expect at most only about $4 \mathrm{~J}$ to appear in the tokamak poloidal field energy. The increase in $I_{p}$ of $1600 \mathrm{~A}$ constituted an increase in poloidal field energy of $0.15 \mathrm{~J}$. This suggests that, in addition to the geometrical efficiency of Eq. (13), we have an electrical efficiency (conversion of capacitor bank energy to spheromak magnetic energy) of somewhat less than $5 \%$. This points out a potential difficulty with this technique for current drive, namely that if the spheromak gun is significantly smaller than the major radius, then the geometrical efficiency will be small $(<1 \%)$. If the geometrical efficiency is then multiplied by the inevitable electrical inefficiency, there will typically be an overall efficiency of $<0.1 \%$.

It is important to verify that spheromak injection has a minimal effect on the other current drive mechanism in the experiment - the Ohmic heating $(\mathrm{OH})$ transformer. We performed a set of experiments to check possible interaction with the external $\mathrm{OH}$ power supply. In these experiments, we monitored the current flowing in the $\mathrm{OH}$ transformer primary during the helicity injection process. These experiments also allow us to account for flux linking the hole in the torus. This is important from the standpoint of helicity conservation for which all the linked flux in our non-simply connected system has to be taken into account. At our typical levels of injected helicity (corresponding to $\Delta \mathrm{I}_{\mathrm{p}} \cong 600 \mathrm{~A}$ ), no effect was observed. However, at somewhat higher levels of injected helicity $\left(\Delta \mathrm{I}_{\mathrm{p}}>1100 \mathrm{~A}\right)$, we observed an additional 160 ampère-turns in the external circuit (0.4 A of current in our 400 turn transformer). There is normally about $10 \mathrm{~A}$ flowing in the $\mathrm{OH}$ circuit to drive a $2 \mathrm{kA}$ tokamak discharge. This corresponds to a 160 A change in the external circuit, compared to $1100 \mathrm{~A}$ flowing in the plasma $(<15 \%)$. This is an important effect; however, it shows that the sudden increase in $I_{p}$ during spheromak injection cannot be attributed to $\Delta \mathrm{I}_{\mathrm{OH}}$.
If the gun flux is disabled and an unmagnetized stream of plasma is injected (Marshall gun injection), we observe the same density increase as with finite $\Phi_{\text {gun }}$ but no $\Delta \mathrm{I}_{\mathrm{p}}$ (Figs 5(e) and (f)). This is expected, since, with $\Phi_{\text {gun }} \cong 0$, the helicity source term vanishes (see Eq. (11)). Similarly, if we fire the gun below the threshold ( $\left(I_{g u n}\right.$ not large enough to overcome $\left.\Phi_{\text {gun }}\right)$, no $\Delta \mathrm{I}_{\mathrm{p}}$ or density increase is observed. In this case, the gun is 'stuffed' and no plasma escapes the gun muzzle. Marshall gun injection has been demonstrated as a viable scheme for tokamak refuelling [27]. However, because an unmagnetized gun fires a stream of plasma, there is an undesirable conducting path for both heat and particles between the gun and the tokamak core. In addition, to the extent that injecting plasma into a tokamak opens flux surfaces and degrades confinement, Marshall gun injection will cause degradation as long as the gun is on. Spheromak injection should cause degradation only for the brief time the spheromak takes to pass through a flux surface (i.e. $\mathrm{t}=\ell_{\mathrm{sph}} / \mathrm{v}_{\mathrm{sph}}$ ).

Figure 6 shows the plasma current and the loop voltage on an expanded time-scale for a right handed spheromak injected into a right handed tokamak. Here again we observe the helicity injection effect only when both discharges have the same sign. Note also that the loop voltage drops as $I_{p}$ increases. This represents the maximum current drive we have observed; $\Delta \mathrm{I}_{\mathrm{p}}=1.6 \mathrm{kA}$. The change in loop voltage is consistent with the change in $\mathrm{I}_{\mathrm{p}} ; \mathrm{V}_{\text {loop }}=\mathrm{L}\left(\mathrm{dI}_{\mathrm{p}} / \mathrm{dt}\right)$ and $\mathrm{L}_{\text {tok }} \cong 0.5 \mu \mathrm{H}$, while $\mathrm{dI} / \mathrm{dt} \cong 1.6 \mathrm{kA} / 10 \mu \mathrm{s}$, which gives a voltage $\left(\Delta \mathrm{V}_{\text {loop }} \cong 80 \mathrm{~V}\right)$ that is within a factor of four of the measured value. It should be noted that this measurement is performed with a flux loop on the

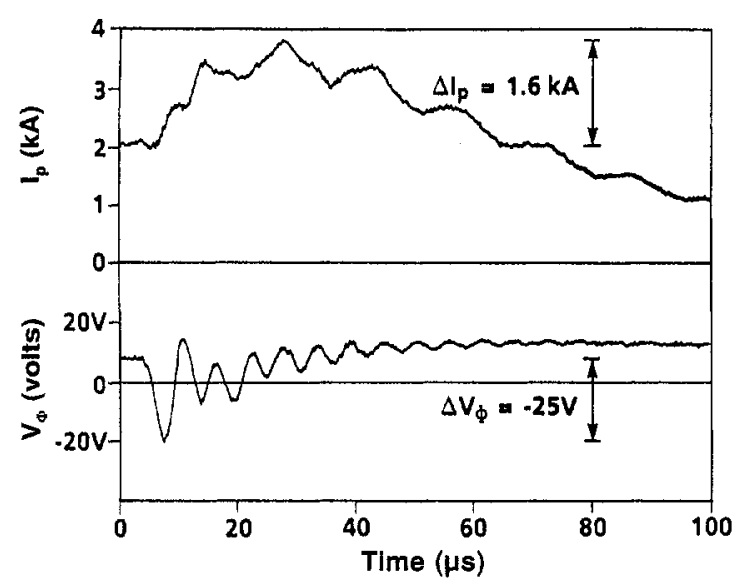

FIG. 6. Plasma current and loop voltage on an expanded timescale. A right handed spheromak is injected into a right handed tokamak, same conditions as in Fig. $5(\mathrm{c}) . \Delta I_{p}=1.6 \mathrm{kA}$ and $\Delta K_{\text {tok }}=10^{-6} \mathrm{~Wb}^{2}$. 
inboard side of the tokamak and is therefore a conservative measure of $V_{\text {loop. }}$. Measurements with an outboard loop gave values typically a factor of two higher than those obtained with the inboard loop.

The oscillations in the $I_{p}$ trace appear to be caused by Alfvénic disturbances (where the oscillation period is the toroidal transit time at the Alfvén velocity). We have observed a magnetic pulse $\left(\tilde{\mathrm{B}}_{\theta}\right)$ moving away from the spheromak injection point at $\cong 15 \mathrm{~cm} / \mu \mathrm{s}$ (which corresponds to a $16 \mu \mathrm{s}$ transit time around the machine). These torsional Alfvén waves can be understood as an expected consequence of the relaxation process depicted schematically in Fig. 3. After the spheromak reconnects with the tokamak fields, the consequent relaxation results in torsional waves moving away from the injection point. We have varied the tokamak toroidal field and found that the velocity of the pulse varies like $B_{\text {tor }}^{1 / 2}$ (rather than varying linearly, as one would expect). At present, we have no satisfactory explanation for this result.

A striking feature of these oscillations is that the oscillation frequency of the loop voltage is nearly twice that of the plasma current. A hypothesis to explain this phenomenon is based on the following argument. The tokamak poloidal flux threading the centre of the torus can be expressed as $\Phi_{\text {pol }}=$ LI, where $\mathrm{L}$ is the tokamak inductance. If we assume that $I_{p}$ varies like $I=I_{0}+\tilde{I} \sin (\omega t)$ (as observed) and that $\mathrm{L}=\mathrm{L}_{0}+\tilde{\mathrm{L}} \sin (\omega \mathrm{t}+\delta)$, then we can express the loop voltage as

$$
\begin{aligned}
& \mathrm{V}_{\text {loop }}=\frac{\mathrm{d}}{\mathrm{dt}}(\mathrm{LI})=\mathrm{L}_{0} \tilde{\mathrm{I}} \omega \cos (\omega \mathrm{t}) \\
& +\tilde{\mathrm{L}} \mathrm{I}_{0} \omega \cos (\omega \mathrm{t}+\delta)+\tilde{\mathrm{L}} \tilde{\mathrm{I}} \omega \sin (2 \omega \mathrm{t}+\delta)
\end{aligned}
$$

The point here is that if both the tokamak plasma current and the inductance are caused to oscillate by the injection of a spheromak, then non-linear coupling gives rise to a term in the loop voltage that has twice the frequency of the oscillation in the current. The magnitude of this term depends on $\tilde{\mathrm{L}}$, where $\tilde{\mathrm{L}}$ can be caused by oscillations in plasma dimension or current profile, or some combination. In order to test this hypothesis, we constructed a plasma position diagnostic (consisting of two loop voltage loops, one inboard and one outboard). Results indicate that there is no gross oscillation of the tokamak plasma major radius upon spheromak injection $\left(\mathrm{L}_{\mathrm{tok}} \cong \mu_{0} \mathrm{R} \ln (8 \mathrm{R} / \mathrm{a})\right.$, so we would expect oscillations in $\mathrm{R}$ to produce the largest oscillations in $\mathrm{L}_{\text {tok }}$ ). However, there appears to be a small oscillation of the plasma out of the horizontal plane (as indicated by phase differences in up-down flux loops) and it is certainly possible that the internal inductance of the tokamak plasma could oscillate because of fluctuations in the current profile.

In Eq. (11), we noted that helicity is created by the magnetized plasma gun at a rate $\dot{\mathrm{K}}=2 \mathrm{~V}_{\text {gun }} \Phi_{\text {gun }}$. It should be noted that $V_{\text {gun }}$ cannot be chosen arbitrarily. The gun voltage is not simply the voltage on the external capacitor bank but rather it depends on the rate at which azimuthal flux is ejected from the gun [22]. In other words, $\mathrm{V}_{\text {gun }}=\dot{\Phi}_{\mathrm{az}}$ where $\dot{\Phi}_{\mathrm{az}}$ is the magnetic flux encircling the inner electrode generated by the gun current. Since $\dot{\Phi}_{\mathrm{az}}$ depends on both the azimuthal magnetic field and the flow velocity (i.e. the Alfvern velocity), $V_{\text {gun }}$ is a non-linear function of the gun current $I_{\text {gun }}$. In addition, if $\Phi_{\text {gun }}$ is too large, then plasma cannot leave the gun and the flow velocity ceases. This is referred to as 'stuffing' the gun and occurs when $\lambda_{\text {gun }}<\lambda_{\text {th }}$ where $\lambda_{\text {gun }}=\mu_{0} \mathrm{I}_{\text {gun }} / \Phi_{\text {gun }}$ and $\lambda_{\text {th }} \cong 4 / r_{\text {gun }}$. We therefore expect that the helicity injected into the tokamak should increase rapidly with increasing $I_{\text {gun }}$ and $\Phi_{\text {gun }}$, but, at sufficiently large $\Phi_{\text {gun }}$, the helicity should vanish. Thus, there is an optimum $\Phi_{\text {gun }}$ at which to operate.

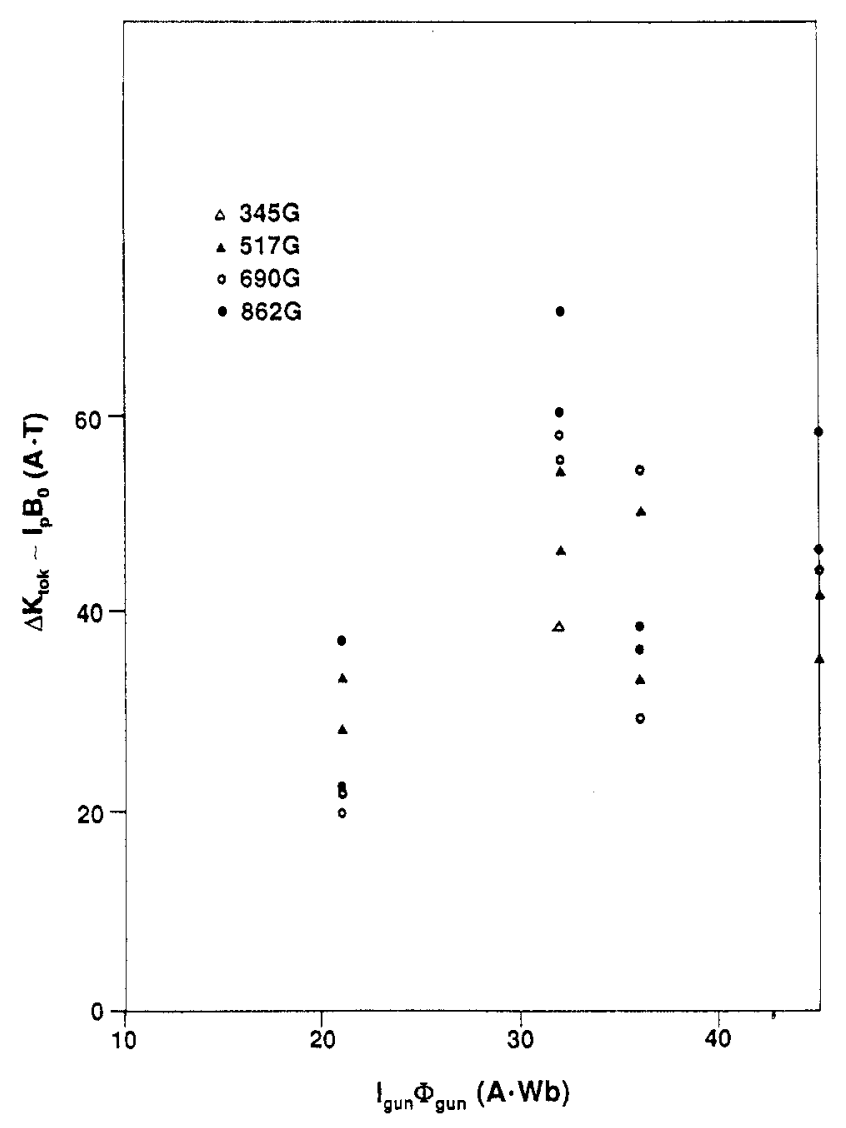

FIG. 7. Increase in tokamak helicity as a function of injected gun helicity. The tokamak helicity is parametrized by $\Delta I_{p} B_{0}$ and the gun helicity by $I_{\text {gun }} \Phi_{\text {gun' }}$ 


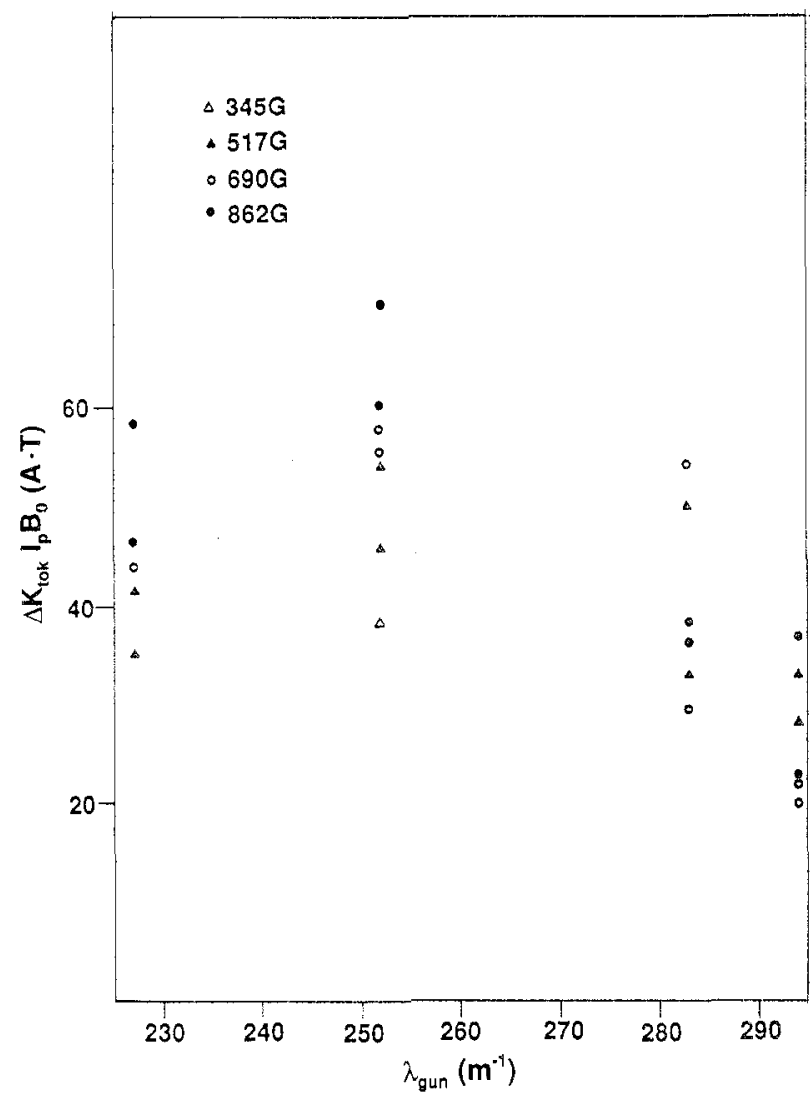

FIG. 8. Increase in tokamak helicity as a function of the gun parameter $\lambda_{\text {gun }}$. Note that there is a threshold at $230 \mathrm{~m}^{-1}$ and the optimum operating point is at $\lambda_{\text {gun }}=250 \mathrm{~m}^{-1}$.

Figure 7 is a plot of the helicity injected into the tokamak as a function of $I_{\text {gun }} \Phi_{\text {gun }}$. The parameter $I_{\text {gun }} \Phi_{\text {gun }}$ is an experimentally convenient measure of the helicity in the gun. We parametrize the increase in tokamak helicity by $\Delta \mathrm{K}_{\text {tok }} \propto \Delta \mathrm{I}_{\mathrm{p}} \mathrm{B}_{0}$, where $\mathrm{B}_{0}$ is the tokamak toroidal field (see Eq. (10)). We have varied $\mathrm{B}_{0}$ by a factor of 2.5 in these scans. The helicity in the gun has been parametrized by $I_{\text {gun }} \Phi_{\text {gun }}$. Both $I_{\text {gun }}$ and $\Phi_{\text {gun }}$ have been varied by a factor of two for these scans. Note that the tokamak helicity initially increases with gun helicity but saturates at high values of $\mathrm{I}_{\text {gun }} \Phi_{\text {gun }}$. This can probably be attributed to both the lowering of plasma flow at high $\Phi_{\text {gun }}$ and the deleterious effects of a high gun current [21]. We have noted that at high currents $\left(I_{\text {gun }}>120 \mathrm{kA}\right)$ the inner electrode becomes pitted and the spheromak lifetime deteriorates.

In Fig. 8, the injected helicity is plotted as a function of the gun parameter, $\lambda_{\text {gun }}=\mu_{0} \mathrm{I}_{\text {gun }} / \Phi_{\text {gun }}$. Note that there is no injected helicity below $\lambda_{\text {gun }} \cong 230 \mathrm{~m}^{-1}$ (the origin of this plot is well to the left of the page). We have determined a gun threshold, $\lambda_{\text {th }} \cong 210 \mathrm{~m}^{-1}$, below which a spheromak is not formed [21]. Therefore, we do not expect to inject helicity into the tokamak until we operate somewhat above this threshold.

There also appears to be an optimum $\lambda_{\text {gun }}$ at which to operate (around $250 \mathrm{~m}^{-1}$ ), below which the gun is 'stuffed' and above which the deleterious effects of high $I_{\text {gun }}$ reduce efficiency. This has also been noted by Barnes et al. [22] in the context of sustaining a spheromak with a large magnetized plasma gun of the CTX experiment. In Fig. 13 of Ref. [22] the CTX gun efficiency $\left(\mathrm{W}_{\mathrm{sph}} / \mathrm{W}_{\mathrm{cap}}\right)$ is plotted as a function of $\lambda_{\text {gun }}$. The plot of Barnes et al. looks similar to our Fig. 8; both exhibit a sharp increase just above $\lambda_{\text {th }}$, a broad maximum and a gradual decrease at large $\lambda_{\text {gun }}$.

\subsection{Refuelling by spheromak injection}

The injected spheromak raises the central tokamak density from about $1 \times 10^{18}$ to $6 \times 10^{18} \mathrm{~m}^{-3}$ (Fig. 2 (g)), as measured by a Langmuir probe at one fourth of the tokamak circumference from the spheromak injection point. Initially, the tokamak has $10^{17}$ plasma particles and the spheromak has $10^{18}$ particles. The increase in tokamak density is consistent with the incorporation of $10^{18}$ spheromak particles. Firing the gas valve alone without firing the gun has no effect on tokamak plasma density or $I_{p}$ since the slowly moving neutral gas does not reach the tokamak vessel until after the discharge is terminated (i.e. the sound velocity of the gas is $\left.\ll v_{\text {Alf }}\right)$. It is therefore clear that tokamak refuelling takes place because of incorporation of spheromak plasma into the tokamak.

The high density/high current discharge persists for less than $100 \mu \mathrm{s}$ (a few particle confinement times). Evidently, this is because the tokamak magnetic pressure is unable to support the additional plasma pressure (i.e. a density or $\beta$ limit is exceeded). After the initial helicity injection effect (increase or decrease depending on the signs), $I_{p}$ decays by about a factor of three. Langmuir probe measurements of the electron temperature (also one fourth of the circumference away from the injection point) show that the tokamak temperature slowly increases (because of Ohmic heating) to $\cong 12 \mathrm{eV}$ just before spheromak injection and then sharply drops to $\cong 4 \mathrm{eV}$ just after injection (Fig. 2(h)). The calculated $\mathrm{L} / \mathrm{R}$ decay time of $\mathrm{I}_{\mathrm{p}}$ due to cooling is $\cong 70 \mu \mathrm{s}$ (for $\mathrm{L}_{\text {tok }} \cong 0.5 \mu \mathrm{H}$ and $\mathrm{R}_{\text {tok }} \cong 7 \mathrm{~m} \Omega$, assuming Spitzer resistivity with $Z=2$ and $T_{e}=4 \mathrm{eV}$ ), which is about the observed value. Measurements of tokamak line emission show an increase in $\mathrm{H}_{\alpha}$ emission of more than a factor of ten upon spheromak injection and little increase in impurity line emission [21]. 
A difficulty in our experiment is that we have little control over either spheromak or tokamak plasma density [21]. We therefore find that $\mathrm{n}_{\mathrm{sph}} / \mathrm{n}_{\text {tok }} \cong 1000$ and the ratio of total particle inventories is $\mathrm{N}_{\mathrm{sph}} / \mathrm{N}_{\mathrm{tok}} \cong 10$. Since spheromak plasma will always be much cooler than tokamak plasma in any spheromak injection refuelling scheme, it is important that $\mathrm{N}_{\text {sph }} / \mathrm{N}_{\text {tok }} \ll 1$ to prevent sudden cooling of the tokamak plasma.

A Langmuir probe array measured the tokamak density profile at four minor radial positions during spheromak injection. The array was located at one fourth of the tokamak circumference away from the spheromak injection point. A typical sequence is depicted in Fig. 9. Before spheromak injection, the tokamak density profile is relatively flat. The injected spheromak increases the tokamak central density (at $50 \mu \mathrm{s}$ ) and sharply peaks the profile. It is clear from this result that spheromak injection fuels the tokamak on axis. The profile then becomes hollow, which is suggestive of a pressure gradient induced interchange instability [28] at $55 \mu \mathrm{s}$. Finally, the profile returns to a less peaked configuration about $60 \mu \mathrm{s}$ after injection. The radial electron temperature profile (also measured with the Langmuir probe array) remains relatively flat. Injection of the spheromak into the empty tokamak vacuum vessel results in a monotonic density profile that decays selfsimilarly in time.

We are currently testing a new spheromak with an acceleration stage [29] to be used with the Phaedrus tokamak at the University of Wisconsin [30]. The accelerator works on the same principle as the Livermore RACE device [25]. The inner and outer electrodes have diameters of $0.08 \mathrm{~m}$ and $0.15 \mathrm{~m}$, respectively. The spheromak accelerator has been designed so that

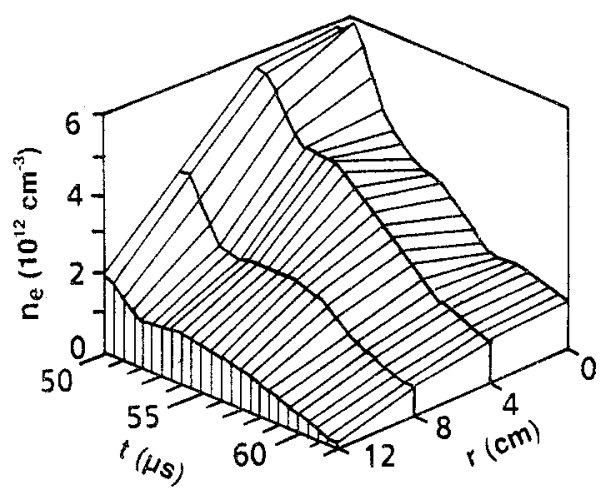

FIG. 9. Tokamak radial density profile upon injection of a spheromak, showing peaking and hollowing indicative of a pressure gradient induced interchange instability. Data obtained with a linear Langmuir probe array.
$\mathrm{N}_{\text {sph }} / \mathrm{N}_{\text {tok }}<1$; in this way we hope to avoid the tokamak cooling problem discussed above. The accelerator stage will increase $\beta_{\mathrm{dyn}}$ as defined in Eq. (16).

\section{DISCUSSION AND SUMMARY}

In order to be a viable refuelling scheme, spheromak injection must replenish the tokamak particle inventory once every particle confinement time. Therefore,

refuelling rate $=\frac{\mathrm{N}_{\text {tok }}}{\mathrm{N}_{\mathrm{sph}}} \frac{1}{\tau_{\mathrm{p}}}$

where $\mathrm{N}$ is the total particle inventory in either the tokamak or the spheromak. Similarly, as a current drive or refluxing scheme, spheromak injection must replenish the tokamak helicity once every tokamak helicity decay time $\tau_{\mathrm{K}}$. Note that for the tokamak, $\tau_{\mathrm{K}} \cong \tau_{\mathrm{W}}$, and $\tau_{\mathrm{W}}$ is roughly half the $L / R$ decay time of the toroidal current, so $\tau_{\mathrm{K}} \cong \tau_{\mathrm{L} / \mathrm{R}} / 2$. If the energy confinement time is much smaller than the helicity decay time, $\tau_{\mathrm{E}} \ll \tau_{\mathrm{K}}$, then the energy must be replenished by other means. We find, therefore,

reflux rate $=\frac{\mathrm{K}_{\text {tok }}}{\mathrm{K}_{\mathrm{sph}}} \frac{1}{\tau_{\mathrm{K}}}$

where $\mathrm{K}_{\text {tok }}$ and $\mathrm{K}_{\mathrm{sph}}$ are the helicity contents of the tokamak and the spheromak, respectively.

Table I presents a comparison of tokamak and spheromak parameters. From these parameters we can assess the viability of spheromak injection for refuelling and refluxing of tokamaks. We can calculate the helicity content of tokamaks and spheromaks from formulas in Section 2.2 and from the particle inventories from the expression $\mathrm{N}_{20}=\mathrm{n}_{20} \times$ volume, where $\mathrm{N}_{20}$ is the total particle inventory in units of $10^{20}$ particles and $n_{20}$ is the particle density in units of $10^{20} \mathrm{~m}^{-3}$. The total energy stored in capacitor banks for the spheromak discharges is also given in the table.

We can see that in the present experiment the spheromak and Caltech's ENCORE tokamak have comparable particle number and helicity content (in fact, $\mathrm{N}_{\mathrm{sph}}=10 \mathrm{~N}_{\text {tok }}$ ). Therefore, in order to refuel and reflux ENCORE, we would need to inject a spheromak once every $\tau_{\mathrm{p}}$ or $\tau_{\mathrm{K}}$, respectively. For the proposed experiment of an accelerated spheromak injected into the Phaedrus tokamak, we can see that a reasonable refuelling rate of about $50 \mathrm{~Hz}$ is required. However, because $\mathrm{K}_{\mathrm{sph}} / \mathrm{K}_{\text {tok }}$ is so small in this case, we find an uncomfortably high refluxing rate of $60 \mathrm{kHz}$. Since the stored capacitor bank energy is $13 \mathrm{~kJ}$ for each spheromak discharge, the average power to reflux Phaedrus with spheromaks of this helicity content is at the 
TABLE I. COMPARISON OF TOKAMAK AND SPHEROMAK PARAMETERS

\begin{tabular}{lcccccccccc}
\hline Machine & $\begin{array}{c}\mathrm{R} \\
(\mathrm{m})\end{array}$ & $\begin{array}{c}\mathrm{a} \\
(\mathrm{m})\end{array}$ & $\mathrm{n}_{20}$ & $\mathrm{~N}_{20}$ & $\begin{array}{c}\mathrm{B} \\
(\mathrm{T})\end{array}$ & $\begin{array}{c}\mathrm{I}_{\mathrm{p}} \\
(\mathrm{MA})\end{array}$ & $\begin{array}{c}\mathrm{K} \\
\left(\mathrm{Wb}^{2}\right)\end{array}$ & $\begin{array}{c}\tau_{\mathrm{K}} \\
\tau_{\mathrm{p}}\end{array}$ & $\begin{array}{c}\mathrm{W} \\
(\mathrm{kJ})\end{array}$ \\
\hline Caltech Spher. & - & 0.04 & 10 & 0.01 & - & 0.1 & $10^{-6}$ & - & - & 4 \\
ENCORE & 0.38 & 0.12 & 0.02 & 0.001 & 0.1 & 0.002 & $10^{-6}$ & $10^{-5}$ & $10^{-5}$ & - \\
Accel. Spher. & - & 0.1 & 100 & 0.5 & - & 0.3 & $10^{-5}$ & - & - & 13 \\
Phaedrus & 0.93 & 0.26 & 0.4 & 0.5 & 1.1 & 0.12 & 0.02 & 0.03 & 0.02 & - \\
CTX & - & 0.67 & 2 & 1 & - & 0.4 & 0.04 & - & - & 300 \\
TFTR & 2.48 & 0.85 & 5 & 200 & 5.2 & 3 & 50 & 1 & 1 & - \\
JET & 2.96 & 1.25 & 4 & 400 & 3.45 & 7.1 & 200 & 1 & 1 & - \\
BPX & 2.57 & 0.79 & 3 & 100 & 9 & 12 & 300 & 5 & 5 & - \\
ITER & 6 & 2.15 & 1.5 & 800 & 4.85 & 22 & 6000 & 10 & 10 & - \\
\hline
\end{tabular}

$500 \mathrm{MW}$ level, but refuelling could take place at the $500 \mathrm{~kW}$ level.

If we consider using the CTX spheromak $[2,31]$ to refuel and reflux the JET tokamak we see that a refuelling rate of $400 \mathrm{~Hz}$ and a refluxing rate of $5 \mathrm{kHz}$ are required. Since the stored energy in the CTX capacitor banks is about $300 \mathrm{~kJ}$, the average power to reflux JET with CTX spheromaks would be $1.5 \mathrm{GW}$. From Eq. (13) the geometrical efficiency for CTX refluxing of JET would be $\epsilon=\mathrm{r}_{\mathrm{gun}} /\left(2 \mathrm{q} \mathrm{R}_{\mathrm{tok}}\right) \cong 0.03$. Since Ohmic current drive on JET requires $\mathrm{P}_{\mathrm{OH}}=\mathrm{I}_{\mathrm{p}} \mathrm{V}_{\text {loop }} \cong 5 \mathrm{MW}$, we would expect a minimum power of $\mathrm{P}_{\mathrm{OH}} / \epsilon \cong 150 \mathrm{MW}$ to be supplied using spheromak injection current drive. Since CTX is about $10 \%$ efficient in making spheromaks, we see that the $1.5 \mathrm{GW}$ estimate above is consistent with this accounting.

A possible solution to the problem of low geometrical efficiency for spheromak injection current drive has been proposed by Jarboe [23]. He suggests that if the spheromak gun were coaxial with the tokamak axis of symmetry, then $r_{\text {gun }} \cong R_{\text {tok }}$. In addition, if the tokamak toroidal field is in the same sense as the azimuthal field in the gun, then the gun impedance can be significantly higher than in conventional operation.

Since there is typically a disparity between the required refuelling and refluxing rates, we cannot expect to both refuel and reflux efficiently. In other words, if CTX were used to refuel JET, then spheromak injection could be expected to drive only $10 \%$ of the current. Conversely, if CTX were used to reflux JET, then the injection process would provide a factor of ten too many particles.
An overriding concern with spheromak injection current drive and refuelling is the effect of spheromak impurities on the tokamak discharge. We had problems with the ablation of the central electrode at high discharge current [21]. In the present experiments, this problem was addressed by coating the central electrode with a refractory material (tungsten or chrome). Future experiments need to address this problem by either using refractory electrode coatings or using a low $\mathrm{Z}$ material to limit the generation of high $Z$ impurities. Since high $\mathrm{Z}$ plasma tends to move more slowly than does the spheromak plasma, it may also be possible to use a magnetic gate to prevent the high $\mathrm{Z}$ material from entering the tokamak discharge.

In summary, small spheromaks injected into Caltech's ENCORE tokamak have been shown to drive plasma current (via helicity injection) as well as substantially increase tokamak density. Central densities have increased by a factor of six, while plasma currents have increased by $30 \%$ initially, followed by a reduction by a factor of three. In order for spheromak refuelling and refluxing of tokamaks to be viable, a number of criteria must be met. First, refluxing requires that both the efficiency $\epsilon$ and the spheromak gun impedance be high. However, the geometrical efficiency

$\epsilon=\frac{\mathrm{W}_{\text {tok }} / \tau_{\mathrm{K}}}{\mathrm{I}_{\text {gun }} \mathrm{V}_{\text {gun }}}=\mathrm{r}_{\text {gun }} / 2 \mathrm{q} \mathrm{R}_{\mathrm{tok}}$

will be small unless (see Ref. [23]) $r_{\text {gun }} \cong R_{\text {tok }}$ and the tokamak operates at low q (presumably, a larger tokamak would be more resilient to spheromak injection and could be operated at lower q). A low electrical 
efficiency further exacerbates the problem. Second, tokamak refuelling requires that the spheromak be small and dense (this requirement may be in conflict with the first one). Finally, it is important for both schemes that the spheromak have low impurity content. It is important to note that unless the refuelling and refluxing rates are comparable, it is difficult to do both effectively.

\section{ACKNOWLEDGEMENTS}

It is a pleasure to acknowledge the technical contributions of $\mathrm{F}$. Cosso and $\mathrm{L}$. Begay, as well as several useful discussions with Dr. C. Barnes, Dr. T. Jarboe, Dr. J. Fernández and Dr. F. Wysocki at Los Alamos, Dr. C. Hartman and Dr. J. Hammer at Livermore and Dr. R. Kirkwood at Caltech.

This work was performed under the auspices of the United States Department of Energy, Grant No. DE-FG03-86ER53232.

\section{REFERENCES}

[1] ROSENBLUTH, M.N., BUSSAC, M.N., Nucl. Fusion 19 (1979) 489.

[2] BARNES, C.W., FERNÁNDEZ, J.C., HENINS, I., et al., Phys. Fluids 29 (1986) 3415.

[3] HARTMAN, C.W., HAMMER, J.H., Phys. Rev. Lett. 48 (1982) 929.

[4] TAYLOR, J.B., Phys. Rev. Lett. 33 (1974) 1139.

[5] TAYLOR, J.B., Rev. Mod. Phys. 58 (1986) 741.

[6] PERKINS, L.J., HO, S.K., HAMMER, J.H., Nucl. Fusion 28 (1988) 1365.

[7] PARKS, P.B., Phys. Rev. Lett. 61 (1988) 1364.

[8] BROWN, M.R., BELLAN, P.M., Phys. Rev. Lett. 64 (1990) 2144.

[9] BROWN, M.R., BELLAN, P.M., Phys. Fluids B 2 (1990) 1306.
[10] MOFFATT, H.K., Magnetic Field Generation in Electrically Conducting Fluids, Cambridge University Press, New York (1978).

[11] BERGER, M.A., FIELD, G.B., J. Fluid Mech. 147 (1984) 133.

[12] WOLTJER, L., Proc. Natl. Acad. Sci. USA 44 (1958) 489.

[13] SCHOENBERG, K.F., MOSES, R.W., Jr., HAGENSON, R.L., Phys. Fluids 27 (1984) 1671.

[14] FRISCH, U., POUQUET, A., LEORAT, J., MAZURE, A., J. Fluid Mech. 68 (1975) 769.

[15] MONTGOMERY, D., TURNER, L., VAHALA, G,, Phys. Fluids 21 (1978) 757.

[16] HAMMER, J.H. (LLNL), personal communication, 1989.

[17] TURNER, W.C., GOLDENBAUM, G.C., GRANNEMAN, E.H.A., et al., Phys. Fluids 26 (1983) 1965.

[18] FINN, J.M., MANHEIMER, W.M., OTT, E., Phys. Fluids 24 (1981) 1336

[19] BONDESON, A., MARKLIN, G., AN, Z.G., CHEN, H.H., LEE, Y.C., LIU, C.S., Phys. Fluids 24 (1981) 1682.

[20] BROWN, M.R., CUTRER, D.M., BELLAN, P.M., Phys. Fluids B 3 (1991) 1198.

[21] BROWN, M.R., BAILEY III, A.D., BELLAN, P.M., J. Appl. Phys. 69 (1991) 6302.

[22] BARNES, C.W., JARBOE, T.R., MARKLIN, G.J., KNOX, S.O., HENINS, I., Phys. Fluids B 2 (1990) 1871.

[23] JARBOE, T.R., Fusion Technol. 15 (1989) 7.

[24] FISCH, N.J., Rev. Mod. Phys. 59 (1987) 175.

[25] HAMMER, J.H., HARTMAN, C.W., EDDLEMAN, J.L., McCLEAN, H.S., Phys. Rev. Lett. 61 (1988) 2843.

[26] ALFVÉN, H., LINDBERG, L., MITLID, P., J. Nucl. Energy, Part C: Plasma Phys. 1 (1960) 116.

[27] LeONARD, A.W., DEXTER, R.N., SPROTT, J.C., Phys. Rev. Lett. 57 (1986) 333.

[28] WYSOCKI, F.J., FERNÁNDEZ, J.C., HENINS, I., JARBOE, T.R., MARKLIN, G.J., Phys. Rev. Lett. 61 (1988) 2457.

[29] BROWN, M.R., BELlAN, P.M., Bull. Am. Phys. Soc. 36 (1991) 2460; BROWN, M.R., BELLAN, P.M., Phys. Fluids B 2 (1990) 1306.

[30] BREUN, R., BROUCHOUS, D., DIEBOLD, D., et al., Fusion Technol. 19 (1991) 1327.

[31] WYSOCKI, F.J., FERNÁNDEZ, J.C., HENINS, I., JARBOE, T.R., MARKLIN, G.J., Phys. Rev. Lett. 65 (1990) 40.

(Manuscript received 17 December 1991

Final manuscript received 31 March 1992) 